\title{
Agôn
}

Revue des arts de la scène

HS 2 | 2014

Mettre en scène le conte

\section{L'absence maternelle, matrice de Cendrillon de Joël Pommerat}

Alexandra von Bomhard

\section{(2) OpenEdition}

1 Journals

Édition électronique

URL : http://journals.openedition.org/agon/3103

DOI : 10.4000/agon.3103

ISSN : 1961-8581

Éditeur

Association Agôn

Référence électronique

Alexandra von Bomhard, "L'absence maternelle, matrice de Cendrillon de Joël Pommerat », Agôn [En ligne], HS 2 | 2014, mis en ligne le 16 septembre 2014, consulté le 10 décembre 2020. URL : http:// journals.openedition.org/agon/3103; DOI : https://doi.org/10.4000/agon.3103

Ce document a été généré automatiquement le 10 décembre 2020.

Association Agôn et les auteurs des articles 


\title{
L'absence maternelle, matrice de Cendrillon de Joël Pommerat
}

\author{
Alexandra von Bomhard
}

1 S'engager dans une réflexion sur l'œuvre de Joël Pommerat pose une première difficulté. L'artiste ne cesse en effet de mettre en évidence son goût pour la complexité, l'incertain, le trouble - pour reprendre le titre de l'ouvrage de Joëlle Gayot ${ }^{1}$. Nous amorçons ici une entreprise paradoxale lors de laquelle nous chercherons à mettre en lumière certains aspects d'un spectacle, sans pour autant nous laisser aveugler par une trop grande clarté.

Ce n'est pas un hasard si la compagnie de l'artiste s'appelle la compagnie Louis Brouillard:

« la Compagnie Louis Brouillard fut fondée en opposition avec le Théâtre du Soleil qu'il côtoyait alors et qui l'impressionnait: "J'eus une réaction un peu potache à cette époque face à ce théâtre où l'on montrait tout, où la vérité et le réel se dévoilaient dans la clarté, tout cela me paraissait suspect et contestable. Et puis, nous nous sommes aperçus que le prénom "Louis" nous ramenait à Louis Lumière, il y avait une opposition entre le montré et le caché, entre la lumière et l'obscurité $»^{2}$.

Nous tenterons donc d'éviter ici un écueil que Joël Pommerat nommerait le «fantasme de domination par le mot, par le texte »:

« Je crois à la complexité. Et je crois même que la complexité est une des définitions de la beauté. La complexité ne me fait pas peur, la contradiction non plus, l'assemblage des contraires m'attire. Nous sommes composés de ces contradictions. En priver le regard, c'est lui dissimuler ce qui fait la beauté de chaque chose de ce monde.

"Louis Brouillard", le nom de ma compagnie vient de ces convictions, j'ai pensé au terme brouillard en opposition avec "clarté", avec "l'esprit français", ce fameux dogme du: "ce qui se conçoit bien s'énonce clairement". Sous-entendu, tout ce qui ne se maîtrise pas par la parole n’a ni intérêt ni réalité. Autrement dit, la parole doit tout cerner. Mais, surtout, il n'y a rien d'autre d'intéressant et d'existant que ce que je peux nommer. Ça démontre un fantasme de domination par le mot, par le texte. Sans doute quelque chose qui a voulu historiquement s'opposer à une domination tyrannique du mystique, du religieux et de l'irrationnel $»^{3}$.

Cette méfiance vis-à-vis des mots est d'ailleurs partagée par la narratrice de Cendrillon : 
«dans l'histoire que je vais raconter, les mots ont failli avoir des conséquences catastrophiques sur la vie d'une très jeune fille. Les mots sont très utiles, mais ils peuvent aussi être très dangereux si on les comprend de travers. Certains mots ont plusieurs sens. D'autres mots se ressemblent tellement qu'on peut les confondre $»^{4}$.

Le sens est sensible chez Pommerat, il se diffuse, mais ne s'appréhende pas comme une évidence. Certes, le discours sur l'œuvre ne saurait se substituer à l'œuvre. Nous souhaiterions néanmoins ne pas anéantir, avec ce travail, la magie du conte, ne pas trop disséquer le spectacle au risque d'en faire un cadavre dans une chambre froide. Pour entrer dans cette œuvre, nous avons choisi d'emprunter le chemin de la famille.

La famille est, traditionnellement, une structure clé du genre théâtral : des sombres destinées des nobles lignées qui hantent les œuvres tragiques, aux foyers bourgeois qui font l'assise de la comédie, cette cellule est au fondement de l'action et des péripéties dramatiques. Pour Joël Pommerat, qui souhaite «raconter la société des hommes et trouver les métaphores qui soient les plus efficaces possibles artistiquement parlant », la famille « est une micro-société, une petite représentation de la société en suffisamment condensé pour que ça puisse être rendu théâtralement $»^{5}$. Cette structure est donc très présente dans son œuvre, plus particulièrement dans ses trois réécritures de contes, Le Petit Chaperon rouge (2004), Pinocchio (2008) et Cendrillon (2011). Dans sa note d'intention à Cendrillon, l'auteur-metteur en scène rappelle en ce sens que :

« d'une certaine façon, les contes relèvent d'un parti pris d'écriture [qu'il a] adopté depuis longtemps, qui consiste à chercher à décrire des faits fictionnels comme s'ils étaient réels. En cherchant une forme de description la plus simple et la plus directe possible. Comme le conte décrit des relations humaines fondamentales, il ne peut pas échapper à la famille. C'est le premier système social. Comme auteur, avant de [s'] ouvrir et de [s'] interroger sur la société entière, [il a] eu besoin d'observer cette petite structure sociale qu'est la famille. Dans les contes, si la famille est si présente, c'est bien parce que tout part de là, que toute destinée humaine y prend sa source. C'est donc important d'y être présent, d'y aller voir, lorsqu'on veut comprendre ou bien raconter l'humanité, d'un point de vue politique par exemple $»^{6}$.

7 De plus dans Cendrillon, la réécriture tire son origine de la famille. Celle-ci naît, paradoxalement, de la mort de la mère et du deuil du personnage éponyme. L'absence maternelle est matrice de la création :

«je me suis intéressé particulièrement à cette histoire quand je me suis rendu compte que tout partait du deuil, de la mort (la mort de la mère de Cendrillon) (...) Pas seulement une histoire d'ascension sociale conditionnée par une bonne moralité qui fait triompher de toutes les épreuves ou une histoire d'amour idéalisée. Mais plutôt une histoire qui parle du désir au sens large : le désir de vie, opposé à son absence. C'est peut-être aussi parce que comme enfant j'aurais aimé qu'on me parle de la mort qu'aujourd'hui je trouve intéressant d'essayer d'en parler aux enfants $»^{7}$.

8 Plus qu'un simple thème, la famille a donc une valeur structurante dans la création. Elle permet en outre de mettre à jour plusieurs enjeux de l'esthétique de la Compagnie Louis Brouillard.

\section{La famille, vecteur de liens entre les personnages}

9 Si la famille intéresse l'auteur, c'est par la simplicité des liens qu'elle induit. Elle est une architecture élémentaire qui permet la construction de la pièce. Mais elle revêt 
également une valeur dramaturgique : en créant des liens entre les personnages, elle est un moteur de l'action théâtrale. Pour Joël Pommerat :

«la famille (au sens parental du terme) est une notion tout simplement pratique dans le processus de création. Son introduction sur le plateau me permet des combinaisons élémentaires. J'ai besoin de cette simplicité pour construire. Besoin de ne pas avoir à expliquer ces liens aux spectateurs. Ils sont donnés. Lorsqu'on entend quelqu'un sur scène qui dit "maman" ou "papa", tout est dit.

Dans les contes, par exemple, il n'est question que de famille. Mais, comme dans mes pièces, ce n'est pas la personne en elle-même qui est importante, c'est ce qu'elle va faire en rapport avec l'autre. Et ce n'est pas cet autre qui est important, c'est la relation, le récit qui va naître et exister entre eux $»^{8}$.

11 Pour analyser les combinaisons élémentaires permises par la cellule familiale, il n'est que de se reporter à la première page, soit à la liste des personnages de Cendrillon. Celle-ci s'articule autour d'un être à partir duquel se déclinent les liens familiaux. Ce procédé est très fréquent chez Joël Pommerat. On le retrouve, par exemple, dans Le Petit Chaperon rouge, Grâce à mes yeux, Au monde.

«Une narratrice dont on n'entend que la voix

un homme qui fait des gestes pendant qu'elle parle

la très jeune fille

la mère

le père

les sœurs : la grande et la petite

la fée

le très jeune prince

le roi

deux gardes $»^{9}$.

12 Dans cette réécriture, un seul personnage porte un nom. Il n'apparaît pas dans la liste,

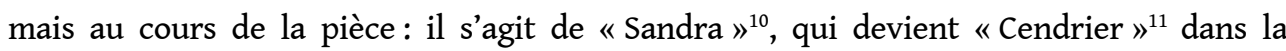
bouche des sœurs, ou de leur mère ${ }^{12}$, puis « Cendrillon » dans celle du très jeune prince ${ }^{13}$. Celle qui est nommée est justement la très jeune fille qui est au cœur de l'histoire, en quête d'identité, en quête d'elle-même. Le spectacle nous raconte aussi peut-être que la difficulté d'être soi réside dans la difficulté de se situer par rapport aux autres. C'est dans la justesse de son rapport à l'autre, aux autres, qu'on peut trouver son identité. François Flahault, philosophe cher à Joël Pommerat, rappelle en ce sens que les contes « insistent sur le fait que nous ne pouvons être tout pour une seule personne, que nous ne saurions être à elle et à elle seule » :

«Ils montrent que la pluralité des liens humains, tout en étant nécessaire et souhaitable, introduit en nous une division que la réalisation de soi, si accomplie soit-elle, ne peut effacer. Comment l'enfant - un être d'abord rivé à la génération qui le précède - parvient-il à se détourner de celle-ci pour prendre sa place d'homme ou de femme parmi ses contemporains ? C'est là une question sur laquelle les contes reviennent sans cesse $\aleph^{14}$.

Mais, si la famille a une valeur structurante dans la création, c'est aussi parce que le conte raconte le cheminement de l'individu au cœur de ses proches.

\section{La famille, lieu d'un cheminement identitaire}

14 Le conte raconte l'histoire de Sandra, mais l'histoire de Sandra croise celle du prince. Si l'on se reporte une nouvelle fois à la liste des personnages, on observe que ce qui ressort, 
c'est la dualité ou le dédoublement : la narratrice est dédoublée par un homme qui fait des gestes. On peut mettre en parallèle la très jeune fille et le très jeune prince. Il y a deux sœurs (la grande, la petite), deux pères (le père, le roi) et deux substituts maternels. L'un est négatif (la marâtre), l'autre, positif (la fée). Il y a même deux gardes! Plus fondamentalement, deux familles sont au cœur du spectacle : la nouvelle famille, qu'on appellerait aujourd'hui famille recomposée, avec la mère, le père, les deux sœurs et la très jeune fille, sur laquelle se centre la première partie. Cette famille en rencontre une autre, dans la deuxième partie, à l'occasion $\mathrm{du}$ bal: la famille royale, qu'on dirait monoparentale, composée du roi et du très jeune prince.

Les parallèles entre ces deux familles sont évidents. La distribution, tout d'abord, nous invite à les rapprocher : ce sont les mêmes acteurs qui interprètent les membres des deux foyers. Ainsi, Caroline Donnelly est à la fois le prince et la sœur la petite, tandis que le père de la très jeune fille est incarné par le même acteur que le père du très jeune prince (Alfredo Cañavate). Mais c'est surtout l'absence de la mère qui leur est commune. La mort de la mère de Cendrillon ouvre la pièce ${ }^{15}$. On apprend celle de la mère du prince de la bouche du roi ${ }^{16}$. Comme le souligne Marion Boudier dans sa postface, la très jeune fille et le très jeune prince «fonctionnent en miroir, leur rencontre les aidant mutuellement à accepter cette perte qui les a, chacun d'une manière différente, coupés du monde $»^{17}$. La mère manquante est une figure que l'on retrouve dans les autres contes réécrits par Joël Pommerat: Pinocchio a un père, «l'homme âgé », mais n'a pas de mère. Quant au Petit Chaperon rouge, elle a bien une mère, mais qui n'est jamais là pour sa fille. L'absence de la mère n'est pas l'apanage des seuls récits pour enfants: dans Au monde, il n'y a plus que le père. La mère de Grâce à mes yeux, défaillante, meurt à la fin de la pièce. D'autres figures de mères fragiles traversent Cet enfant...

16 Ce vide est au cœur du conte et à l'origine de la quête identitaire de Cendrillon. Il s'accompagne, dans les deux cas, d'un rapport trouble à la parole qui génère de la douleur. Pour Cendrillon, il s'agit d'un malentendu, au sens premier du terme : elle a mal entendu les mots de sa mère. Voici ce que la très jeune fille comprend des paroles inaudibles prononcées par celle-ci : « Ma petite fille, quand je ne serai plus là il ne faudra jamais que tu cesses de penser à moi. Tant que tu penseras à moi tout le temps sans jamais m'oublier, je resterai en vie quelque part $\aleph^{18}$. Ce malentendu, obsédant, est à l'origine de l'histoire: «Ce qui est certain, c'est que cette histoire n'aurait pas été la même si la très jeune fille avait entendu parfaitement ce que sa mère lui avait dit $»^{19}$. $\mathrm{Ce}$ n'est qu'à la fin du spectacle que l'on entend les vraies paroles de la mère, qui furent, en réalité : «Ma chérie... Si tu es malheureuse, pour te donner du courage, pense à moi... mais n'oublie jamais, si tu penses à moi fais-le toujours avec le sourire $»^{20}$.

Pour le très jeune prince, la mort de la mère est accompagnée d'un mensonge, ce qui est le pire selon Françoise Dolto: "si quelqu'un de la famille meurt, il est important de ne jamais priver l'enfant de la nouvelle de cette mort », car « ne pas lui dire, c'est l'exclure de la communauté des parlants $»^{21}$, et par la même, rendre le travail de deuil impossible. Or, le père du très jeune prince a justement choisi de ne pas dire la vérité à son fils : "Comme vous le savez évidemment, sa mère est morte quand il avait cinq ans. Depuis ce jour, pour lui épargner une trop grande souffrance, je lui raconte que sa mère est partie en voyage et qu'elle a du mal à rentrer à cause d'incessantes grèves des transports. Mais chaque soir, je dois trouver un nouveau mensonge pour justifier qu'elle ne l'appelle pas et ça c'est terrible $»^{22}$. C'est de la bouche de Cendrillon que le prince apprend la mort de sa mère : 
«tu penses pas des fois qu'on est en train de te raconter des histoires avec cette histoire? [...]

Ce que je veux dire... c'est que je crois savoir que ce soir ta maman elle va pas t'appeler... et demain non plus... et dans une semaine non plus [...]

Parce que ta maman, parce que ta mère, son coeur il bat plus... depuis dix ans... depuis dix ans elle est morte ta mère... en fait ta mère est morte voilà $\rrbracket^{23}$.

La très jeune fille et le très jeune prince se rejoignent alors dans le travail de deuil qu'ils doivent accomplir :

« LE TRES JEUNE PRINCE : Tu aimerais ça moi que je te dise que ta mère est morte?

LA TRES JEUNE FILLE : Ben tu pourrais... tu pourrais me le dire... Parce que c'est la vérité, ma mère est morte et tu sais moi aussi faut que j'arrête je crois de me raconter des histoires, me raconter qu'elle va peut-être revenir un jour ma mère, si je pense à elle continuellement par exemple, non ! Elle est morte et c'est comme ça ! Elle va pas revenir ma mère ! Et elle est morte ! Comme la tienne ! Et rien ne pourra y changer ? Non rien. ${ }^{24}$.

En dépit de ces points de convergence, le conte ne s'achève pas par la fondation d'une nouvelle famille. Le couple formé par la très jeune fille et le très jeune prince n'est pas éternel. Seuls restent les mots qu'ils s'envoient ${ }^{25}$. En lieu et place du traditionnel « ils se marièrent et eurent beaucoup d'enfants ", le spectateur est laissé dans une certaine incertitude. L'histoire est en effet relayée par la voix de la narratrice, dont on ne connaît pas l'identité exacte: «Je vais vous raconter une histoire d'il y a très longtemps. Tellement longtemps que je ne me rappelle plus si dans cette histoire c'est de moi qu'il s'agit ou bien de quelqu'un d'autre $»^{26} /$ "Comme je vous l'ai dit pour commencer, je ne me rappelle plus si cette histoire est la mienne ou bien l'histoire de quelqu'un d'autre ${ }^{27}$. S'agit-il de la voix de Sandra? Toujours est-il que la famille est au centre de l'histoire narrée par cette voix. Loin d'être une simple thématique, elle structure la pièce, revêt une force dramaturgique. Plus essentiellement, il convient de dépasser à présent le texte (qui n'est que la trace laissée par le spectacle pour Pommerat ${ }^{28}$ ) pour s'intéresser aux enjeux esthétiques que véhicule la famille.

\section{La famille dessine l'espace}

L'élément marquant de la scénographie est bien entendu la maison en verre de la famille de la marâtre. Comme le souligne Daniel Loayza, Joël Pommerat est parti d'intuitions matérielles pour sa réécriture : « la cendre, le verre - et comme un étrange flottement de lumière sombrement tangible, un espace clos qui hésiterait entre l'excès et le manque de fenêtres, tel que sait lui donner corps Eric Soyer ${ }^{29}$, scénographe de la compagnie Louis Brouillard. Cette maison en verre est peut-être une discrète résurgence de la pantoufle de verre (vair) que l'on trouve dans la version de Perrault. C'est surtout un espace à la fois clos (par sa matérialité) et ouvert (par sa transparence), à la fois familier (c'est une maison, elle devrait être le foyer, le lieu où se retrouve la famille) et inquiétant (loin d'être chaleureux, le verre impose un espace froid ; de plus, les oiseaux viennent s'écraser contre ses vitres - vitres que Sandra doit par ailleurs nettoyer). Il s'agit d'un espace trouble, complexe, propice à l'assemblage des contraires cher à la Compagnie Louis Brouillard. Eric Soyer rappelle en ce sens que l'un des enjeux majeurs de la scénographie dans les spectacles de Joël Pommerat est de «mettre l'espace en mouvement » : « il s'agit de fabriquer une boîte dans laquelle on oublie le théâtre et où tout devient possible au niveau de l'imaginaire. On s'attache à faire disparaître les limites du lieu où l'on inscrit la 
représentation. C'est une boîte de lumière qui permet de créer des tensions dans l'espace $30 »$.

21 Cette maison, qui a par ailleurs une force comique (lorsque Sandra et son père cherchent à y entrer et que l'on entend les commentaires désobligeants des deux soeurs), dit aussi la difficulté des relations entre les êtres. La transparence donne l'illusion d'être ensemble, de partager un même espace, mais en réalité, les êtres se voient séparés par un mur de verre infranchissable. Les courses-poursuites dans les couloirs ${ }^{31}$, par le mouvement des corps qui s'évitent, donnent à voir une famille éclatée, tandis que la cave sombre dans laquelle Sandra est reléguée traduit de manière très évidente l'ostracisme dont celle-ci est victime. Espace ambigu, qui se situe entre intérieur et extérieur, la maison de verre nous révèle quelque chose de ses habitants. La belle-mère dit au sujet de cet endroit : « on voit mal à l'intérieur de l'extérieur $~_{32}$. Cette formule nous rappelle, entre autres, le jeunisme du personnage qui cherche toujours à paraître moins que son âge. Mais cette maison nous apprend-elle, plus fondamentalement, quelque chose de l'être humain ? Cet intérieur qui se dérobe derrière l'apparence extérieure, ne vient-il pas aussi concrétiser une des recherches de Joël Pommerat pour qui «dire le monde et sa réalité, c'est entremêler l'intérieur et l'extérieur ${ }^{33}$ ?

La maison de verre se présente en outre comme un espace inquiétant et étouffant. Les oiseaux ne cessent de s'écraser contre ses parois invisibles qui laissent malgré tout entrevoir une végétation luxuriante. Comme le souligne Christophe Triau, la «nature semble contenue derrière les vitres comme une menace qu'il s'agit de refouler $»^{34}$. L'espace familial est marqué par une artificialité froide et oppressante. Au contraire, Sandra rappelle que sa mère, elle, aimait les oiseaux ${ }^{35}$. Elle imagine également qu'elle «restera en vie dans un endroit secret invisible tenu par des oiseaux ${ }^{36}$. Ce motif des oiseaux (discrète réminiscence des volatiles qui aident Cendrillon dans la version des frères Grimm ?) permet l'envol vers un ailleurs, celui du souvenir et de l'imagination, seul espace possible de la mère défunte.

Le palais apparait dans la deuxième partie, c'est le lieu de la famille royale. On reste toujours au seuil de cet espace, on n'assiste pas au bal (du moins pas visuellement: ce n'est qu'à travers le son que la soirée est évoquée, par la musique et les rires moqueurs des autres invités à l'arrivée de la famille ridiculisée par le choix de ses costumes $d^{\prime}$ ' époque ${ }^{37}$ ). La rencontre du prince et de la belle-mère, celle du prince et de Cendrillon ont lieu devant la porte, sans doute parce que la réécriture de Joël Pommerat laisse de côté l'ascension sociale au coeur du conte original.

Que ce soit au niveau des relations entre les êtres, au niveau de l'espace ou de la dynamique de l'histoire, la famille est au cœur de la pièce. On analysera à présent comment la question de la famille porte la trace des convictions esthétiques fondamentales de Joël Pommerat et de sa compagnie.

\section{La famille, reflet de l'univers artistique de Joël Pommerat}

25 La mort de la mère de Cendrillon est à l'origine de la réécriture de Pommerat. C'est elle qui ouvre la pièce et qui hante continuellement les pensées de la très jeune fille ${ }^{38}$. De fait, Sandra ne cesse d'évoquer sa mère à travers un album photo : 
«Tenez, ça c'est une photo de ma mère quand elle était jeune. Elle avait les cheveux courts à cette période [...]

C'est une photo de ma mère et de mon père quand ils étaient venus me voir à un spectacle de fin d'année à la maternelle [...]

Ça c'est une photo un peu particulière, un peu cochonne « olé ! Olé » comme on dit entre mon père et ma mère $[. ..] »^{39}$

dégoûtant ma mère était sortie je sais pas pourquoi je m'étais mise à le faire, ma mère en rentrant m'avait dit [...] ma mère elle aimait bien les oiseaux [...] $»^{40}$ ) et à travers sa montre qui sonne toutes les cinq minutes (sur l'air de «Ah vous dirais-je maman ») pour lui rappeler de penser à sa mère. En somme, Sandra est obnubilée par sa mère, tout au long de la pièce (tout comme le très jeune prince, qui ne parle que de sa mère depuis dix ans). C'est dire que l'absente est omniprésente dans le spectacle. Cet être n'est plus là, mais sa présence n'en reste pas moins évidente. Voilà un des enjeux majeurs de la recherche artistique de Joël Pommerat :

«Les choses qui m'intéressent valent pour ce qu'elles sont capables de révéler d'autre, de différent, voire de contraire, c'est leur profondeur qui m'intéresse. Je vise quelque chose derrière l'action, les mots, la situation. Quelque chose qu'on ne doit pas pouvoir désigner simplement, quelque chose qui doit apparaître, quelque chose qui doit s'immiscer, se glisser entre les lignes des gestes et des phrases prononcées, comme une réalité fantôme bien plus présente, bien plus forte sous cette forme que si elle était désignée par le texte ou par le jeu des interprètes, par leurs intentions affirmées, soulignées.

Une réalité fantôme comme ces membres fantômes, ces jambes ou ces bras qui ont été amputés et dont la présence continue à se faire ressentir » ${ }^{41}$.

mère de la très jeune fille, absente et présente à la fois, correspond à cette réalité fantôme que l'auteur metteur en scène appelle de ses vœux. Cette figure incertaine, transitoire, est au cœur de l'art de Joël Pommerat et de sa compagnie, qui, ensemble, cherchent à capter quelque chose du réel fugace et transitoire, ce quelque chose qui relève de «l'équilibre précaire $»^{42}$.

Dans le spectacle, cette absence/ présence est rendue sensible par un accessoire (faut-il dire un costume ?) : la robe de la mère de la très jeune fille. En effet, on apprend que Sandra dort avec cette robe. Une nuit, la belle-mère surprend le père apporter en cachette à Sandra cette robe, sorte d'objet transitionnel :

«LE PERE : Chez nous, elle avait l'habitude de l'avoir avec elle dans sa chambre, ça l'aide à dormir! C'est des trucs de gosses ça! C'est pas grave! ça va lui passer! Après elle nous laissera tranquilles tu vois! Elle fera sa petite vie avec sa mère... avec la robe de sa mère et voilà ! C'est pour nous que je fais ça en réalité! Pour qu'on se retrouve nous! Nous deux ! »33.

Le lapsus du père montre que l'équivalence entre l'être et le vêtement de la mère ne se fait pas seulement dans l'esprit de Sandra. D'ailleurs, la belle-mère s'écrie ensuite: «Jamais ton ex-femme ne viendra dans ma maison! / LE PERE : Mais elle est morte. / LA BELLE-MERE : Ça m'est égal ! il est hors de question qu'elle habite chez moi ! c'est tout !» ${ }^{44}$.

On sait l'importance du costume pour la compagnie. Isabelle Deffin (costumière de la troupe) considère que son travail consiste à trouver le vêtement en parfaite adéquation avec l'identité et la personnalité des personnages ${ }^{45}$. Cette quête artistique, qui consiste à trouver presque une équivalence entre le personnage et le costume est en germes dans le texte : la robe de la mère est, d'une certaine manière, la mère. En un sens, cet accessoire 
vient donner corps à la mère morte. Elle donne à voir de manière concrète l'absente, la réalité fantôme, pour le plus grand trouble du spectateur. On touche là à un point essentiel des convictions et de l'univers artistique de Joël Pommerat. Le réel ne se limite pas au rationnel, «cette tension entre réalité réelle et réalité perçue ou imaginée $~^{46}$ traverse toute son œuvre :

« les choses », nous dit-il « sont composées de ce qu'elles sont et de l'imaginaire qui les accompagne. La réalité se situe aussi dans la tête $»^{47}$

«Je souhaitais donner corps, non pas au réel ou à l'action, mais à l'imaginaire »"8. «Pour toucher à la réalité humaine, il ne faut pas choisir entre le dedans et le dehors mais admettre l'entremêlement des deux ${ }^{49}$.

La robe de la mère n'est pas seulement robe, vêtement. Si, elle est dans l'esprit de Sandra, la mère, alors cette robe est robe et mère à la fois, elle est à la fois vide et pleine d'une absente / présente.

Cette présence fantomatique ne cesse d'être réactivée par un accessoire: la montre de Cendrillon. Celle-ci a une vertu comique. Sa sonnerie (une «musique entêtante $»^{50}$ nous dit le texte, qui devient "Ah vous dirais-je maman» dans le spectacle), gênante, interrompt les uns et les autres. Elle rappelle également à Cendrillon la promesse faite à sa mère : se souvenir d'elle à chaque instant.

«Avant la très jeune fille aimait beaucoup laisser son imagination prendre possession de ses pensées. Mais maintenant tout ça, c'était bien fini. Elle devait concentrer son esprit sur un seul et unique sujet : sa mère... seulement sur sa mère. Les premiers temps, c'était simple. Mais après quelques mois, un jour, il arriva qu'elle oublie. Il arriva qu'elle oublie pendant quelques instants. Elle eut très peur. Le lendemain, elle demanda à son père de lui acheter une montre. La plus grosse possible. Equipée d'une sonnerie comme d'un réveil. Pour contrôler le temps ${ }^{51}$.

Le problème de cette montre, c'est qu'elle coupe Cendrillon du présent. Elle l'empêche d'être avec les autres. C'est la question du rapport au temps qui est intéressante : Sandra croit empêcher sa mère de tomber dans la vraie mort, elle croit pouvoir la retenir dans un éternel présent, qu'elle tente de saisir à travers cette montre ridicule. Cette quête vaine, impossible, nous rappelle une quête essentielle de Joël Pommerat, de ses acteurs et de nous, ses spectateurs. Citant La pensée des contes de François Flahault, l'artiste rappelle en effet que dans l'acte de conter avec un public, il n'existe pas de séparation, d'opposition et de distinction entre "l'événement d'être ensemble » et la fable. La définition du conte, c'est cette recherche d'être ensemble. C'est le plaisir d'être relié à quelqu'un au moyen de l'imaginaire dans un temps donné.

\footnotetext{
« Dès mes premiers spectacles, j'ai su que mes histoires étaient des prétextes à créer de l'instant ? Or, qu'est-ce que l'instant ? L'instant, c'est du lien [...]

je crois que lorsque les spectateurs parlent d'intensité au sujet de mes spectacles, c'est parce qu'ils ont précisément ressenti que les personnages s'inscrivaient dans le même temps et le même espace qu'eux.

Cet espace n'est pas coupé, il n'y a pas de quatrième mur.

Tous, spectateurs, acteurs, personnages, se retrouvent dans un instant du présent commun. Ce n'est pas n'importe quel instant : c'est du temps présent, c'est-à-dire du temps qui meurt à chaque instant. Il évolue, sans cesse, il passe, il survient et il cesse, se renouvelle, disparaît à nouveau ${ }^{52}$.
}

Ainsi, Sandra, avec sa montre, nous donne une grande leçon de théâtre, et de vie. De la même manière que nous éteindrons notre portable au début de la pièce, Sandra, à la fin, éteint sa montre : elle a accompli son travail de deuil, acceptant de vivre au présent, c'està-dire dans un temps qui meurt à chaque instant... 


\section{NOTES}

1. GAYOT Joëlle/POMMERAT Joël, Joël Pommerat, troubles, Arles, Actes Sud, 2009.

2. POMMERAT Joël, cité in Joël Pommerat ou le corps fantôme, Flore Lefebvre des Noëttes, Master II d'Etudes Théâtrales, septembre 2007, pp. 74-75.

3. POMMERAT Joël, in GAYOT Joëlle/POMMERAT Joël, Joël Pommerat, troubles, op. cit., p. 37.

4. POMMERAT Joël, Cendrillon, Arles, Actes Sud, Babel, 2013, pp. 9-10.

5. POMMERAT Joël, URL : http://www.arte.tv/fr/L-actualite-du-festival/1282266.html

6. POMMERAT Joël, Note d'intention, in Dossier d'accompagnement au spectacle "Cendrillon », URL : http://www.theatre-odeon.eu/sites/default/files/pj/da_cendrillon.pdf, pp. 18-19.

7. Idem.

8. POMMERAT Joël, in GAYOT Joëlle/POMMERAT Joël, Joël Pommerat, troubles, op.cit., p. 68.

9. POMMERAT Joël, Cendrillon, Arles, Actes Sud, Babel, 2013, p. 6.

10. Ibid., I, 5, p. 17.

11. Ibid., I, 11, p. 40.

12. Ibid., I, 11, p. 44.

13. Ibid., II, 12, p. 104.

14. FLAHAULT François, L'Interprétation des contes, Denoël, Paris, 1988, p. 9.

15. POMMERAT Joël, Cendrillon, op. cit, I, 3, p. 13.

16. Ibid., II, 9, p. 89.

17. BOUDIER Marion, Postface, in Cendrillon, Arles, Actes Sud, Babel, 2013, p. 135.

18. POMMERAT Joël, Cendrillon, op. cit, I, 2, p. 12.

19. Idem.

20. POMMERAT Joël, Cendrillon, op. cit, II, 14, p. 112.

21. DOLTO Françoise, Lorsque l'enfant paraît, tome I, Paris, Editions du Seuil, « Points ", n P595, p. 100.

22. POMMERAT Joël, Cendrillon, op. cit, II, 9, p. 89.

23. Ibid., II, 12, pp. 100-101.

24. Ibid., II, 12, pp. 101-102.

25. Ibid., II, 15, p. 112.

26. Ibid., I, 1, p. 9.

27. Ibid., II, 14, p. 111.

28. POMMERAT Joël, in GAYOT Joëlle/POMMERAT Joël, Joël Pommerat, troubles, op. cit., p. 19.

29. LOAYZA Daniel, in Dossier d'accompagnement au spectacle "Cendrillon", http://www.theatreodeon.eu/sites/default/files/pj/da_cendrillon.pdf, pp. 48-49.

30. SOYER Eric, in Dossier d'accompagnement au spectacle «Cendrillon », p. 20.

31. POMMERAT Joël, Cendrillon, op. cit, II, 10, p. 90.

32. Ibid., I, 4, p. 15.

33. POMMERAT Joël, in GAYOT Joëlle/POMMERAT Joël, Joël Pommerat, troubles, op.cit., p. 46.

34. TRIAU Christophe, Joël Pommerat. Cendrillon, Paris, CNDP, « Arts au singulier THEATRE », 2013, p. 36 .

35. POMMERAT Joël, Cendrillon, op. cit, I, 10, p. 36.

36. POMMERAT Joël, Cendrillon, op. cit, I, 2, p. 12.

37. Ibid., II, 2, p. 66.

38. Ibid., I, 3, p. 13.

39. Ibid., I, 5, pp. 17-22. 
40. Ibid., I, 10, pp. 32-38.

41. POMMERAT Joël, Théâtres en présence, Actes Sud Papier, collection " Apprendre", Arles, 2007,

p. 27.

42. Ibid., p. 29.

43. POMMERAT Joël, Cendrillon, op. cit, I, 8, p. 30.

44. Idem.

45. DEFFIN Isabelle, in Dossier d'accompagnement au spectacle "Cendrillon ", URL: http:// www.theatre-odeon.eu/sites/default/files/pj/da_cendrillon.pdf, p. 21.

46. BOUDIER Marion, Postface, in Cendrillon, Arles, Actes Sud, Babel, 2013, p. 117.

47. POMMERAT Joël, in GAYOT Joëlle/POMMERAT Joël, Joël Pommerat, troubles, op. cit., pp. 68-69.

48. Ibid., p. 46.

49. Ibid., p. 48.

50. POMMERAT Joël, Cendrillon, op. cit, I, 5, p. 18.

51. Ibid. I, 3, p. 13.

52. POMMERAT Joël, in GAYOT Joëlle/POMMERAT Joël, Joël Pommerat, troubles, op. cit., pp. 60-61.

INDEX

Mots-clés : Pommerat (Joël), Cendrillon 